\title{
Time Domain Implementation of Transmitting Boundaries in ABAQUS for Discrete Soil-structure Interaction Systems
}

\author{
J. Rama Raju Patchamatla \\ Department of Civil Engineering, \\ Graphic Era (Deemed to be University), Dehradun, Uttarakhand, India. \\ E-mail: pjramaraju@gmail.com \\ P. K. Emani \\ Department of Civil Engineering, \\ Graphic Era (Deemed to be University), Dehradun, Uttarakhand, India. \\ Corresponding author: pk.emani@gmail.com
}

(Received August 10, 2019; Accepted November 27, 2019)

\begin{abstract}
Soil-structure-interaction (SSI) analyses are essential to evaluate the seismic performance of important structures before finalizing their structural design. SSI under seismic condition involves much more complex interaction with soil compared to the dynamic loads having source on the structure. Seismic SSI analysis requires due consideration of sitespecific and structure-specific properties to estimate the actual ground motion (scattered motion) experienced at the base of the structure, and subsequently the effects of the scattered motion on the structure. Most challenging aspect of seismic SSI analysis is to implement transmitting boundaries that absorb the artificial reflections of stress waves at the truncated interface of the finite and infinite domains, while allowing the seismic waves to enter the finite domain. In this paper, the time domain implementation of seismic analysis of a soil-structure system is presented using classical discrete models of structure and interactive force boundary conditions for soil. These models represent typical SSI systems- a single Degree of Freedom (DOF) of a spherical cavity with mass attached to its wall, a two DOF system consisting of a mass attached by a nonlinear spring to a semi-infinite rod on elastic foundation, and a three DOF system with additional DOFs for modelling the structural stiffness and damping. The convolution integral representing the force boundary condition on the truncated interface, is evaluated interactively using UAMP user-subroutine in ABAQUS and applied as concentrated forces at the interface (truncated interface) nodes of the bounded domain or generalized-structure domain. The verification problems presented in the paper show the satisfactory performance of the developed MATLAB code and ABAQUS implementation with FORTRAN user-subroutines. The classical phenomena associated with the dynamic soil-structure systems are discussed through the present work.
\end{abstract}

Keywords- Soil-structure interaction, Seismic analysis, Transmitting boundaries, ABAQUS, UAMP subroutine.

\section{Introduction}

The accuracy, and thus the usefulness, of a dynamic Soil-Structure Interaction (SSI) analysis depend on the ability to model the boundary at the truncated interface of the finite geometry (bounded domain) and the semi-infinite geometries (unbounded domain). The frequency dependence of the stiffness of the unbounded domain along with the stress waves and their multiple reflections makes the modelling of this domain the most challenging part. Although classical solutions of this SSI problem are obtained either in the frequency domain (Emani and Maheshwari, 2009; Spyrakos and Xu, 2003; Wolf, 1985), or hybrid domain (Emani and Maheshwari, 2010; Maheshwari and Emani, 2015), the dynamic analysis is intuitively done only in time domain using the numerical integration of the equations of motion. The displacement boundary conditions are highly inappropriate at the truncated interface since such boundaries cause considerable reflection of waves leading to chaotic results in the finite geometry (Kramer, 
International Journal of Mathematical, Engineering and Management Sciences

Vol. 5, No. 3, 447-462, 2020

https://doi.org/10.33889/IJMEMS.2020.5.3.037

1996). So, a time domain implementation of the (interaction-) force boundary condition is needed motion (Sarkar and Maheshwari, 2012; Solberg et al., 2016; Wegner et al., 2005; Wolf and Song, 1996). That method which can integrate with the well-developed state-of-the-art finite element (FE) solvers like ABAQUS will be of special significance. Recently, researchers are using user subroutines to achieve customization of FE solvers for SSI analysis (Poul and Zerva, 2018; Wang and Yang, 2013). In the following section the results for three representative soil-structure systems- one modelled as a single DOF, another as 2DOF and one other system modelled as 3 DOF are presented. The implementation in all three cases is done using UAMP user subroutine in ABAQUS.

\section{Formulations of System and Method of Analysis}

Classically a SSI system is modelled as a finite domain with the effect of the truncated soil accounted through an interactive force applied at the truncated interface called as "soil structure interface". Basic equation of motion for such a system in time domain is given by Eq. (1) (Wolf, 1988)

$\left[\begin{array}{ll}{\left[M_{s s}\right]} & {\left[M_{s b}\right]} \\ {\left[M_{b s}\right]} & {\left[M_{b b}\right]}\end{array}\right]\left\{\begin{array}{l}\left\{\ddot{u}_{s}^{t}(t)\right\} \\ \left\{\ddot{u}_{b}^{t}(t)\right\}\end{array}\right\}+\left\{\begin{array}{c}\left\{P_{s}(t)\right\} \\ \left\{P_{b}(t)\right\}\end{array}\right\}=\left\{\begin{array}{c}\{0\} \\ \left\{R_{b}(t)\right\}\end{array}\right\}$

where $[M]$ is the partitioned form of mass matrix at the structure nodes (with subscript ss) and at the interface nodes (subscript bb) of the finite domain, $\{P(t)\}$ is the partitioned internal force vector as a function of time $t$. The soil-structure interaction force vector $\left\{R_{b}(t)\right\}$ is obtained from convolution integral of unit displacement impulse response (or dynamic stiffness matrix in time domain) $\left[S_{b b}(t)\right]$ of the truncated unbounded domain, and the relative motion at the interface $\left(\left\{u_{b}^{t}(\tau)\right\}-\left\{u_{b}^{g}(\tau)\right\}\right)$, as in Eq. (2)

$\left\{R_{b}(t)\right\}=\int_{0}^{t}\left(\left[S_{b b}(t-\tau)\right]\left(\left\{u_{b}^{t}(\tau)\right\}-\left\{u_{b}^{g}(\tau)\right\}\right) d \tau\right.$

In time domain, the dynamic stiffness matrix of truncated unbounded domain has a general form given in Eq. (3)

$\left[S_{b b}(t)\right]=\left[K_{b b}^{g}\right] \delta(t)+\left[C_{b b}^{g}\right] \dot{\delta}(t)+\left[S_{r}, g b(t)\right]$

Where $\left[k_{b b}^{g}\right],\left[c_{b b}^{g}\right]$ are the constant stiffness and damping matrices components of the asymptotic (or high frequency) values of the dynamic stiffness matrix, and $\left[S_{r, b b}^{g}(t)\right]$ is the remaining or regular part.

On substituting Eq. (3) in Eq. (2)

$$
\begin{gathered}
R_{b}(t)=\left[K_{b b}^{g}\right]\left(\left\{u_{b}^{t}(t)\right\}-\left\{u_{b}^{g}(t)\right\}\right)+\left[C_{b b}^{g}\right]\left(\left\{\dot{u}_{b}^{t}(t)\right\}-\left\{\dot{u}_{b}^{g}(t)\right\}\right) \\
+\int_{0}^{t}\left[S_{r},{ }_{b b}^{g}(t-\tau)\left(\left\{u_{b}^{t}(\tau)\right\}-\left\{u_{b}^{g}(\tau)\right\}\right) d \tau\right]
\end{gathered}
$$

After time discretization as per Newmark- time integration scheme with implicit algorithm, the Eq. (4), at time step $t=n \Delta t$ becomes 
International Journal of Mathematical, Engineering and Management Sciences

Vol. 5, No. 3, 447-462, 2020

https://doi.org/10.33889/IJMEMS.2020.5.3.037

$$
\begin{aligned}
R_{b}(t)=\left[K_{b b}^{g}\right] & \left(\left\{u_{b}^{t}\right\}_{n}-\left\{u_{b}^{g}\right\}_{n}\right)+\left[C_{b b}^{g}\right]\left(\left\{\dot{u}_{b}^{t}\right\}_{n}-\left\{\dot{u}_{b}^{g}\right\}_{n}\right) \\
& +\int_{0}^{n \Delta t}\left[S_{r}^{g}, b b\right.
\end{aligned}
$$

The basic equations of Newmark time integration method for determining total displacements and velocities of the soil-structure interaction nodes ' $b$ ' at current time step are

$$
\begin{aligned}
& \left\{u_{b}^{t}\right\}_{n}=\left\{u_{b}^{t}\right\}_{n-1}+\Delta t\left\{\dot{u}_{b}^{t}\right\}_{n-1}+\left(\frac{1}{2}-\beta\right) \Delta t^{2}\left\{\ddot{u}_{b}^{t}\right\}_{n-1}+\beta \Delta t^{2}\left\{\ddot{u}_{b}^{t}\right\}_{n} \\
& \left\{\dot{u}_{b}^{t}\right\}_{n}=\left\{\dot{u}_{b}^{t}\right\}_{n-1}+(1-\gamma) \Delta t\left\{\ddot{u}_{b}^{t}\right\}_{n-1}+\gamma \Delta t\left\{\ddot{u}_{b}^{t}\right\}_{n}
\end{aligned}
$$

Using Eq. (6), (7), the velocities $\left\{\dot{u}_{b}^{t}\right\}_{n}$ and accelerations $\left\{\ddot{u}_{b}^{t}\right\}_{n}$ at current step can be expressed in terms of displacements $\left\{u_{b}^{t}\right\}_{n}$ at $t=n \Delta t$.

$$
\begin{aligned}
\left\{\ddot{u}_{b}^{t}\right\}_{n}= & \frac{1}{\beta \Delta t^{2}}\left[\left\{u_{b}^{t}\right\}_{n}-\left\{u_{b}^{t}\right\}_{n-1}-\Delta t\left\{\dot{u}_{b}^{t}\right\}_{n-1}-\left(\frac{1}{2}-\beta\right) \Delta t^{2}\left\{\ddot{u}_{b}^{t}\right\}_{n-1}\right] \\
\left\{\dot{u}_{b}^{t}\right\}_{n}=\left\{\dot{u}_{b}^{t}\right\}_{n-1} & +(1-\gamma) \Delta t\left\{\ddot{u}_{b}^{t}\right\}_{n-1} \\
& \quad+\frac{\gamma}{\beta \Delta t}\left[\left\{u_{b}^{t}\right\}_{n}-\left\{u_{b}^{t}\right\}_{n-1}-\Delta t\left\{\dot{u}_{b}^{t}\right\}_{n-1}-\left(\frac{1}{2}-\beta\right) \Delta t^{2}\left\{\ddot{u}_{b}^{t}\right\}_{n-1}\right]
\end{aligned}
$$

On substituting Eq. (8) and Eq. (9) in Eq. (5)

$$
\begin{aligned}
R_{b}(t)=\left[K_{b b}^{g}\right] & \left(\left\{u_{b}^{t}\right\}_{n}-\left\{u_{b}^{g}\right\}_{n}\right) \\
& +\left[C_{b b}^{g}\right]\left(\left\{\dot{u}_{b}^{t}\right\}_{n-1}+(1-\gamma) \Delta t\left\{\ddot{u}_{b}^{t}\right\}_{n-1}\right. \\
& +\frac{\gamma}{\beta \Delta t}\left[\left\{u_{b}^{t}\right\}_{n}-\left\{u_{b}^{t}\right\}_{n-1}-\Delta t\left\{\dot{u}_{b}^{t}\right\}_{n-1}-\left(\frac{1}{2}-\beta\right) \Delta t^{2}\left\{\ddot{u}_{b}^{t}\right\}_{n-1}\right] \\
& \left.-\left\{\dot{u}_{b}^{g}\right\}_{n}\right)+\int_{0}^{n \Delta t}\left[S_{r}{ }_{b b}^{g}(t-\tau)\left(\left\{u_{b}^{t}(\tau)\right\}-\left\{u_{b}^{g}(\tau)\right\}\right) d \tau\right]
\end{aligned}
$$

The convolution integral in Eq. (10) can be evaluated by using dynamic stiffness matrix of frequency domain through Fourier Transform operations, as follows

$$
\begin{aligned}
& \int_{0}^{n \Delta t}\left[S_{r},{ }_{b b}^{g}(t-\tau)\left(\left\{u_{b}^{t}(\tau)\right\}-\left\{u_{b}^{g}(\tau)\right\}\right) d \tau\right]=R_{1}+R_{2} \\
& R_{1}=\int_{0}^{(n-1) \Delta t}\left[S_{r},{ }_{b b}^{g}(t-\tau)\right]\left(\left\{u_{b}^{t}(\tau)\right\}-\left\{u_{b}^{g}(\tau)\right\}\right) \\
& =\frac{1}{T} \sum_{j=-\infty}^{\infty}\left[S_{r, b b}^{g}\left(\omega_{j}\right)\right]\left(\int_{0}^{(n-1) \Delta t}\left(\left\{u_{b}^{t}(\tau)\right\}-\left\{u_{b}^{g}(\tau)\right\}\right) e^{-i \omega_{j} \tau} d \tau\right) e^{i \omega_{j} t}
\end{aligned}
$$


International Journal of Mathematical, Engineering and Management Sciences

Vol. 5, No. 3, 447-462, 2020

https://doi.org/10.33889/IJMEMS.2020.5.3.037

$$
\begin{aligned}
R_{2}=\int_{(n-1) \Delta t}^{n \Delta t}[ & \left.S_{r, b b}^{g}(t-\tau)\right]\left(\left\{u_{b}^{t}(\tau)\right\}-\left\{u_{b}^{g}(\tau)\right\}\right) d \tau \\
& =\left[S_{r, b b}^{g}\right]_{1}^{*}\left(\left\{u_{b}^{t}\right\}_{n-1}-\left\{u_{b}^{g}\right\}_{n-1}\right)+\left[S_{r, b b}^{g}\right]_{0}\left(\left\{u_{b}^{t}\right\}_{n}-\left\{u_{b}^{g}\right\}_{n}\right)
\end{aligned}
$$

where

$\left[S_{r, b b}^{g}\right]_{1}^{*}=\int_{0}^{\Delta t}\left(1-\frac{\tau^{\prime}}{\Delta t}\right)\left[S_{r, b b}^{g}\left(\Delta t-\tau^{\prime}\right)\right] d \tau^{\prime}$

$\left[S_{r, b b}^{g}\right]_{0}=\int_{0}^{\Delta t}\left(\frac{\tau^{\prime}}{\Delta t}\right)\left[S_{r, b b}^{g}\left(\Delta t-\tau^{\prime}\right)\right] d \tau^{\prime}$

On substituting back Eq. (14) and Eq. (15) in Eq. (13), then Eq. (13) in Eq. (11), then Eq. (12) in Eq. (11), then Eq. (11) in Eq. (25), the formulation can be reduced to

$\left[\widetilde{K}_{b b}\right]\left\{u_{b}^{t}\right\}_{n}=\left\{\tilde{p}_{b}\right\}$

where the effective stiffness $\left[\widetilde{K}_{b b}\right]$ and effective force $\left\{\tilde{p}_{b}\right\}$ are given in Eq. (17) and Eq. (18)

$$
\begin{aligned}
& {\left[\widetilde{K}_{b b}\right]=\frac{1}{\beta \Delta t^{2}}\left[M_{b b}\right]+\left[K_{b b}^{g}\right]+\frac{\gamma}{\beta \Delta t}\left[C_{b b}^{g}\right]+\left[S_{r, b b}^{g}\right]_{0}} \\
& \left\{\tilde{p}_{b}\right\}=\frac{1}{\beta \Delta t^{2}}\left[M_{b b}\right]\left(\left\{u_{b}^{t}\right\}_{n-1}+\Delta t\left\{\dot{u}_{b}^{t}\right\}_{n-1}+\left(\frac{1}{2}-\beta\right) \Delta t^{2}\left\{\ddot{u}_{b}^{t}\right\}_{n-1}\right)+\left[K_{b b}^{g}\right]\left(\left\{u_{b}^{g}\right\}_{n}\right)- \\
& {\left[C_{b b}^{g}\right]\left(\left\{\dot{u}_{b}^{t}\right\}_{n-1}+(1-\gamma) \Delta t\left\{\ddot{u}_{b}^{t}\right\}_{n-1}+\frac{\gamma}{\beta \Delta t}\left[-\left\{u_{b}^{t}\right\}_{n-1}-\Delta t\left\{\dot{u}_{b}^{t}\right\}_{n-1}-\left(\frac{1}{2}-\right.\right.\right.} \\
& \left.\left.\beta) \Delta t^{2}\left\{\ddot{u}_{b}^{t}\right\}_{n-1}\right]-\left\{\dot{u}_{b}^{g}\right\}_{n}\right)+\left[S_{r, b b}^{g}\right]_{0}\left\{u_{b}^{g}\right\}_{n}-R_{1}-\left[S_{r, b b}^{g}\right]_{1}^{*}\left(\left\{u_{b}^{t}\right\}_{n-1}-\left\{u_{b}^{g}\right\}_{n-1}\right)
\end{aligned}
$$

Alternative to the process described from Eq. (11) to Eq. (14), the convolution integral can also be evaluated using displacement impulse response (or dynamic stiffness in time domain) as follows

$$
\begin{aligned}
& \int_{0}^{n \Delta t}\left[S_{r, b b}^{g}(t-\tau)\left(\left\{u_{b}^{t}(\tau)\right\}-\left\{u_{b}^{g}(\tau)\right\}\right) d \tau\right]=R_{1}+R_{2} \\
& R_{1}=\sum_{i=1}^{n-1}\left[S_{r, b b}^{g}\right]_{n-i}\left(\left\{u_{b}^{t}\right\}_{i}-\left\{u_{b}^{g}\right\}_{i}\right) \\
& R_{2}=\left[S_{r, b b}^{g}\right]_{0}\left(\left\{u_{b}^{t}\right\}_{n}-\left\{u_{b}^{g}\right\}_{n}\right)
\end{aligned}
$$

where $\left[S_{r, b b}^{g}\right]_{n-i}$ is given by Eq. (22) and Eq. (23), while $\left[S_{r, b b}^{g}\right]_{0}$ is similar to Eq. (15).

$$
\left[S_{r, b b}^{g}\right]_{n-i}=I_{1}+I_{2}
$$


International Journal of Mathematical, Engineering and Management Sciences

Vol. 5, No. 3, 447-462, 2020

https://doi.org/10.33889/IJMEMS.2020.5.3.037

$I_{1}=\int_{0}^{\Delta t} \frac{\tau^{\prime}}{\Delta t}\left[S_{r, b b}^{g}\left((n-i+1) \Delta t-\tau^{\prime}\right)\right] d \tau^{\prime}$

$I_{2}=\int_{0}^{\Delta t}\left(1-\frac{\tau^{\prime}}{\Delta t}\right)\left[S_{r, b b}^{g}\left((n-i) \Delta t-\tau^{\prime}\right)\right] d \tau^{\prime}$

On substituting back into the equation of motion Eq. (1) the formulation can be reduced to Eq. (16) and can be solved for total displacements. Using Eq. (7) and Eq. (8), the total velocities and accelerations are also evaluated subsequently.

\section{Flow Chart}

The flow chart in

Figure 1 depicts the implementation of the above equations in a programming language. In the present work, MATLAB is used for this purpose.

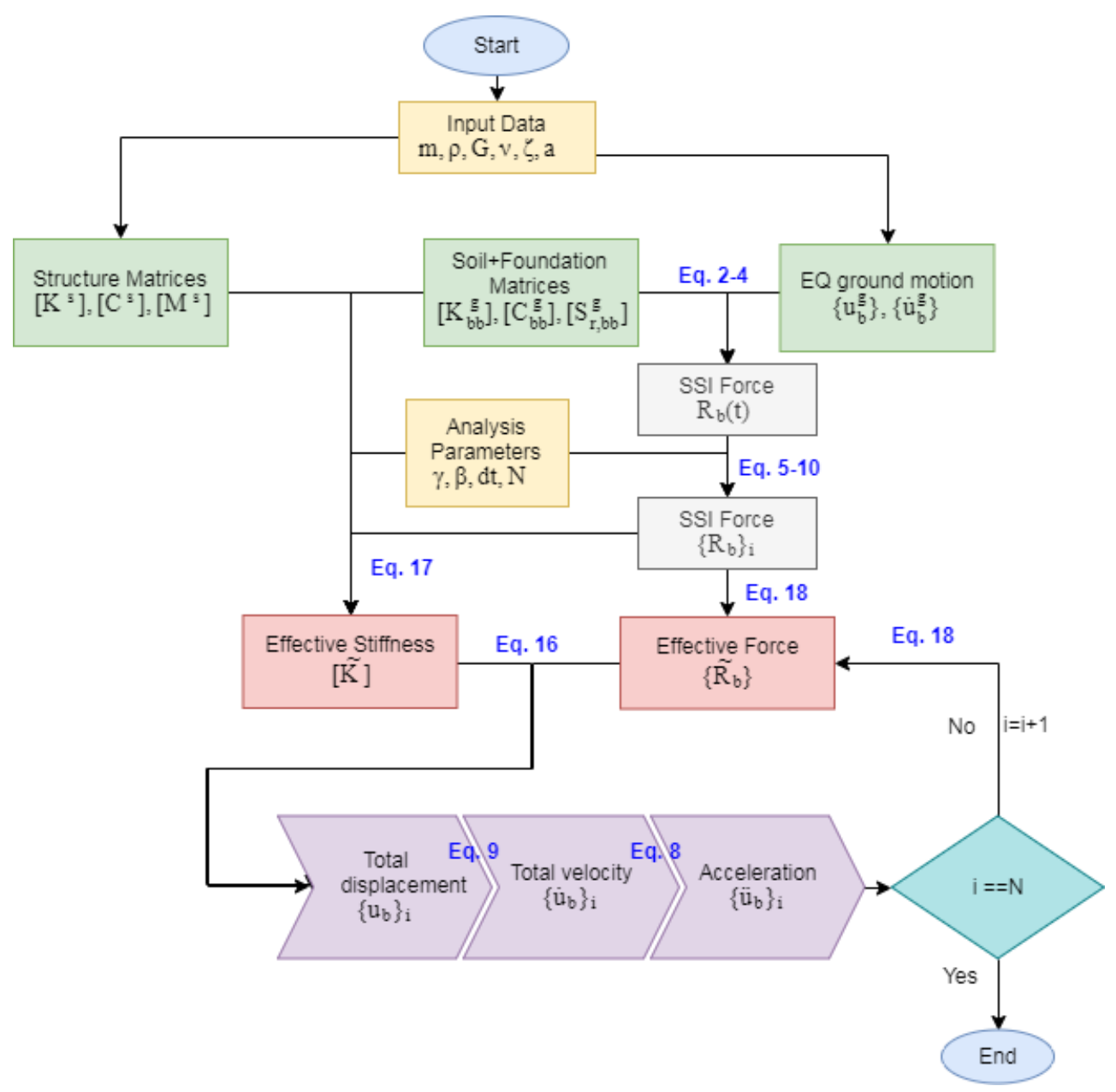

Figure 1. Flow chart of analysis of SSI system for EQ base excitation 
International Journal of Mathematical, Engineering and Management Sciences

Vol. 5, No. 3, 447-462, 2020

https://doi.org/10.33889/IJMEMS.2020.5.3.037

\section{Implementation in ABAQUS}

ABAQUS is general purpose Finite Element software by Dessault systems. The procedure described in the previous section can also be implemented in ABAQUS using an implicit dynamic analysis step. In this case, the time discretization (involving the Newmark's integration parameters, namely, $\gamma$ and $\beta$ ) is internally performed by ABAQUS. Thus, only the terms involving convolution integral and the ground motion are to be considered in the interaction forces, which shall act on a fixed base system. This results in the solution in total displacements, velocities and accelerations.

The calculation of convolution integral in ABAQUS needs an interactive procedure at each increment of the dynamic step. This is because the convolution integral term of the applied interaction forces at any increment depends on the displacements, velocities and accelerations at all the previous steps. For this, sensors are created for displacements, velocities and accelerations at the SSI nodes. Using the sensor data at each step, the convolution terms and thus the total interaction forces on SSI nodes are calculated by a user subroutine UAMP. The UAMP subroutine is coded in FORTRAN 77 for calculating the interaction forces at each time increment. These forces when applied to the fixed base structure give the total displacements, velocities and accelerations at all nodes of the model. These steps are summarized in

Table 1.

Table 1. Summary of steps involved in time domain implementation

\begin{tabular}{|c|c|c|c|c|}
\hline \multirow[t]{2}{*}{ Description of the Step } & \multirow{2}{*}{$\begin{array}{l}\text { Implementation } \\
\text { Platform }\end{array}$} & \multicolumn{3}{|c|}{ Equation numbers } \\
\hline & & SDOF & 2DOF & 3DOF \\
\hline Modelling the structure without any foundation (or support) & ABAQUS & Eq. (1), (25) & Eq. (1) & Eq. (1) \\
\hline $\begin{array}{l}\text { Evaluating or importing the dynamic stiffness coefficients of } \\
\text { the foundation in frequency domain }\end{array}$ & MATLAB & \multirow{2}{*}{ Eq. (29) } & Eq. (35-(36) & Eq. (40),(41) \\
\hline $\begin{array}{l}\text { Resolving the dynamic stiffness coefficients into singular and } \\
\text { regular parts }\end{array}$ & MATLAB & & Eq. (37) & -- \\
\hline $\begin{array}{l}\text { Transforming the regular part into time domain using inverse } \\
\text { Fourier transform }\end{array}$ & MATLAB. & $\begin{array}{l}\text { Eq. (3), (26)- } \\
(28)\end{array}$ & Eq. (38),(39) & IFFT \\
\hline \multirow{2}{*}{$\begin{array}{l}\text { Evaluating the continuous convolution integral of regular part } \\
\text { between the time steps for all time steps (either analytically or } \\
\text { numerically) }\end{array}$} & \multirow[t]{2}{*}{ MATLAB } & \multicolumn{3}{|c|}{ Eq. (14), (15) or Eq. (22), (23), (24). } \\
\hline & & $\begin{array}{l}\text { Eq. } \quad(30), \\
(31)\end{array}$ & \multicolumn{2}{|c|}{ Numerical integration } \\
\hline $\begin{array}{l}\text { The total displacements of base DOF at all previous time steps } \\
\text { (state data) are obtained from sensor data }\end{array}$ & ABAQUS & $\begin{array}{l}\text { Using state va } \\
\text { steps }\end{array}$ & ables svars(n) & previous time \\
\hline \multirow[t]{2}{*}{$\begin{array}{l}\text { Performing discrete convolution (summation for all steps till the } \\
\text { time step previous to current step) to get the amplitude of } \\
\text { interaction forces to be applied at the base nodes. }\end{array}$} & \multirow[t]{2}{*}{$\begin{array}{l}\text { FORTRAN } \\
\text { subroutine } \\
\text { UAMP }\end{array}$} & \multicolumn{3}{|c|}{ Eq. (19), (20), (21) } \\
\hline & & Eq. (32) & Numerically & \\
\hline \multirow[t]{2}{*}{$\begin{array}{l}\text { Adding the contribution of known terms of the convolution } \\
\text { integral term (in the current time step) to the interaction forces }\end{array}$} & \multirow{2}{*}{$\begin{array}{l}\text { FORTRAN } \\
\text { subroutine } \\
\text { UAMP }\end{array}$} & \multicolumn{3}{|c|}{ Eq. (18) } \\
\hline & & Eq. (32) & Numerically & \\
\hline \multirow[t]{2}{*}{$\begin{array}{l}\text { Adding the contribution of unknown terms to the LHS of the } \\
\text { equation of motion. This leads to additional stiffness and } \\
\text { damping to the system at the base. These values are assigned to } \\
\text { the connector at the base }\end{array}$} & \multirow[t]{2}{*}{ ABAQUS } & \multicolumn{3}{|c|}{$\begin{array}{l}\text { Eq. (17). In case of ABAQUS solver the first } \\
\text { term of Eq. (17) is implicitly considered, } \\
\text { while the other three terms form spring and } \\
\text { dashpot values. }\end{array}$} \\
\hline & & Eq. (32) & \multicolumn{2}{|l|}{ Numerically } \\
\hline $\begin{array}{l}\text { Solving the equation of motion to get the accelerations, total } \\
\text { displacements, and total velocities at all DOF of the model }\end{array}$ & $\begin{array}{l}\text { ABAQUS/ } \\
\text { MATLAB }\end{array}$ & \multicolumn{3}{|c|}{$\begin{array}{l}\text { Eq. (16) when using MATLAB for solution or } \\
\text { Eq. (1) when using ABAQUS for solution }\end{array}$} \\
\hline
\end{tabular}


International Journal of Mathematical, Engineering and Management Sciences

Vol. 5, No. 3, 447-462, 2020

https://doi.org/10.33889/IJMEMS.2020.5.3.037

\section{Analysis of SDOF Soil-Structure Interaction (SSI) System}

In the simplest possible system representing general features of SSI, a mass $m$ is attached to a spherical cavity of radius $a$ and subjected to symmetric (radial) stress waves. The medium has a shear modulus of $G$, mass density of $\rho$ and Poisson's ratio of $\nu$. Such a system can be modelled as a SDOF system with mass supported by frequency dependent springs as shown in

(a)

(b)

Figure 2. This SDOF system is analysed (using the procedure formulated in previous section) under symmetric ground excitation $u_{b}^{g}$ to obtain the total displacement response history $u^{t}$ of the mass.

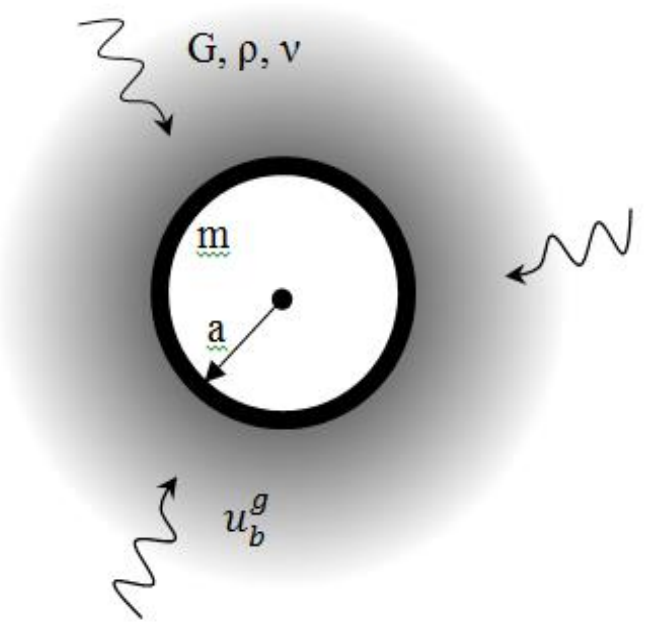

(a)

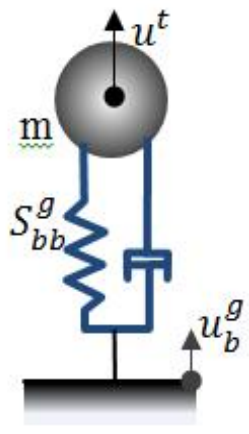

(b)

Figure 2. (a) and (b) Mass attached to spherical cavity and its idealized SDOF system

In the present SDOF SSI system, namely the mass attached to spherical cavity, basic equation of motion is given by Eq. (25)

$m \ddot{u}_{b}^{t}(t)+R_{b}(t)=0$

Where the soil-structure interaction force $R_{b}(t)$ is obtained from convolution integral of Unit displacement impulse response (or dynamic stiffness coefficient in time domain) $S_{b b}(t)$ and the relative motion of the base $\left(\left\{u_{b}^{t}(\tau)\right\}-\left\{u_{b}^{g}(\tau)\right\}\right)$, as in Eq. (2). The components of $S_{b b}(t)$ in this case are given by Eq. (26) to Eq. (28).

$\left[k_{b b}^{g}\right]=K\left(1-\frac{c_{p}^{2}}{4 c_{s}^{2}}\right)$ 
International Journal of Mathematical, Engineering and Management Sciences

Vol. 5, No. 3, 447-462, 2020

https://doi.org/10.33889/IJMEMS.2020.5.3.037

$\left[c_{b b}^{g}\right]=K \frac{a}{c_{p}}\left(\frac{c_{p}^{2}}{4 c_{s}^{2}}\right)$

$\left[S_{r}, \stackrel{g}{g}(t)\right]=K \frac{c_{p}}{a}\left(\frac{c_{p}^{2}}{4 c_{S}^{2}} e^{-\frac{t c_{p}}{a}}\right)$

Here $c_{p}, c_{S}$ represent the $\mathrm{P}$-wave and $\mathrm{S}$-wave velocities in the medium respectively, and $K$ is the static stiffness value of the spherical cavity. The regular part of dynamic stiffness in frequency domain is given by Eq. (29)

$\left[S_{r, b b}^{g}\left(\omega_{j}\right)\right]=K \frac{c_{p}^{2}}{4 c_{S}^{2}} \frac{1}{1+\frac{i \omega a}{c_{p}}}$

Eq. (14) and Eq. (15) can be used to derive the following expressions for $\left[S_{r, b b}^{g}\right]_{1}^{*}$ and $\left[S_{r, b b}^{g}\right]_{0}$

$\left[S_{r, b b}^{g}\right]_{1}^{*}=K \frac{c_{p}^{2}}{4 c_{s}^{2}} \frac{1}{\Delta t}\left[\frac{a}{c_{p}}-\left(\Delta t+\frac{a}{c_{p}}\right) e^{-\frac{\Delta t c_{p}}{a}}\right]$

$\left[S_{r, b b}^{g}\right]_{0}=K \frac{c_{p}^{2}}{4 c_{s}^{2}} \frac{1}{\Delta t}\left[\Delta t-\frac{a}{c_{p}}\left(1-e^{-\frac{\Delta t c_{p}}{a}}\right)\right]$

Eq. (16) for the present case becomes Eq. (32)

$$
\begin{aligned}
m \frac{1}{\beta \Delta t^{2}}\left[\left\{u_{b}^{t}\right\}_{n}\right. & \left.-\left\{u_{b}^{t}\right\}_{n-1}-\Delta t\left\{\dot{u}_{b}^{t}\right\}_{n-1}-\left(\frac{1}{2}-\beta\right) \Delta t^{2}\left\{\ddot{u}_{b}^{t}\right\}_{n-1}\right] \\
& +\left[K_{b b}^{g}\right]\left(\left\{u_{b}^{t}\right\}_{n}-\left\{u_{b}^{g}\right\}_{n}\right) \\
& +\left[C_{b b}^{g}\right]\left(\left\{\dot{u}_{b}^{t}\right\}_{n-1}+(1-\gamma) \Delta t\left\{\ddot{u}_{b}^{t}\right\}_{n-1}\right. \\
& +\frac{\gamma}{\beta \Delta t}\left[\left\{u_{b}^{t}\right\}_{n}-\left\{u_{b}^{t}\right\}_{n-1}-\Delta t\left\{\dot{u}_{b}^{t}\right\}_{n-1}-\left(\frac{1}{2}-\beta\right) \Delta t^{2}\left\{\ddot{u}_{b}^{t}\right\}_{n-1}\right] \\
& \left.-\left\{\dot{u}_{b}^{g}\right\}_{n}\right)+R_{1}+\left[S_{r, b b}^{g}\right]_{1}^{*}\left(\left\{u_{b}^{t}\right\}_{n-1}-\left\{u_{b}^{g}\right\}_{n-1}\right) \\
& +\left[S_{r, b b}^{g}\right]_{0}\left(\left\{u_{b}^{t}\right\}_{n}-\left\{u_{b}^{g}\right\}_{n}\right)=0
\end{aligned}
$$

For the alternative procedure described by Eq. (19) to Eq. (24), the following expressions are derived

$$
\begin{aligned}
& I_{1}=K \frac{c_{p}^{2}}{4 c_{s}^{2}} \frac{1}{\Delta t}\left[e^{\Delta t(-n+i) \frac{c_{p}}{a}}\left(\Delta t-\frac{a}{c_{p}}\right)+\frac{a}{c_{p}} e^{-n^{\prime} \Delta t \frac{c_{p}}{a}}\right] \\
& I_{2}=K \frac{c_{p}^{2}}{4 c_{s}^{2}}\left[e^{\Delta t(-n+i) \frac{c_{p}}{a}}\left(\frac{1}{\Delta t} \frac{a}{c_{p}} e^{\Delta t \frac{c_{p}}{a}}-1-\frac{1}{\Delta t} \frac{a}{c_{p}}\right)\right]
\end{aligned}
$$


International Journal of Mathematical, Engineering and Management Sciences

Vol. 5, No. 3, 447-462, 2020

https://doi.org/10.33889/IJMEMS.2020.5.3.037

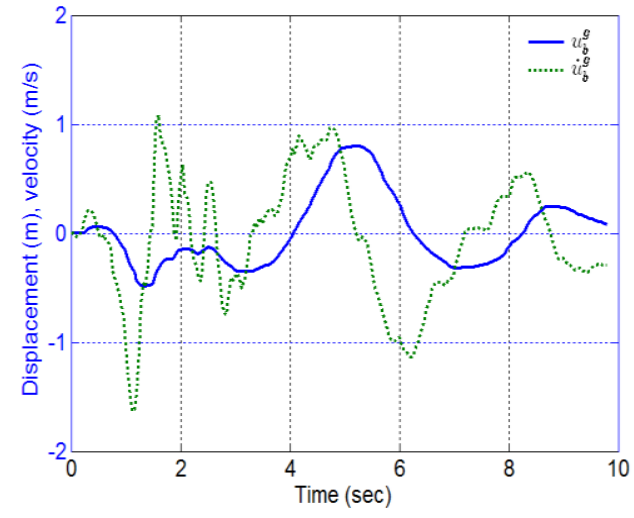

(a)

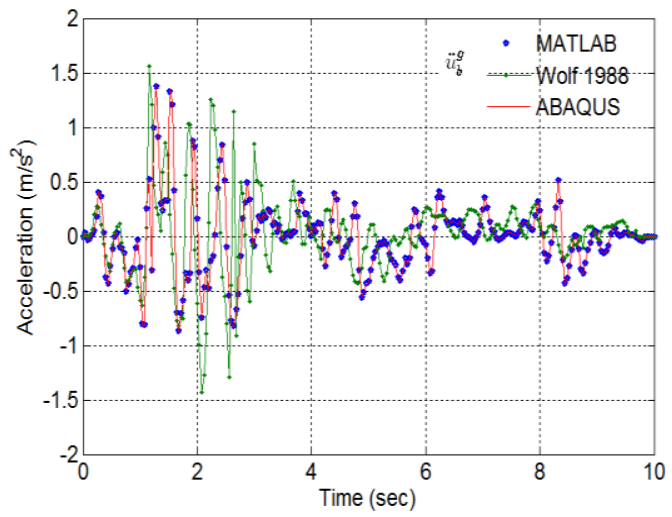

(b)

Figure 3. (a) Input ground motion, (b) Acceleration response of the mass attached to spherical cavity under symmetric P-wave loading

The verification of the developed code and its implementation is checked for an artificial earthquake used by Wolf (1988). The ground motion $\left\{u_{b}^{g}(t)\right\},\left\{\dot{u}_{b}^{g}(t)\right\}$ and the response in terms of the total acceleration of the mass are shown in the Figure 3. The ABAQUS implementation matches the result from MATLAB programme indicating that there are no hidden errors in implementation. The minor discrepancy in the result compared to the result reported by Wolf, 1988 is the sight numerical errors in digitizing the input ground motion used by Wolf, 1988.

The formulation and its implementation in MATLAB and ABAQUS are, thus, verified using the single DOF system of spherical cavity. The same procedure can be applied for any general soilstructure system. The soil modelling can be either continuum or discrete.

\section{Analysis of 2DOF System}

The selected system is a mass $(m)$ connected by a nonlinear spring (of stiffness $k_{s}$ and to a semiinfinite rod on elastic foundation (Figure 4 (a)). The mass and non-linear spring represents the structure and the semi-infinite rod represents the foundation-soil. The dynamic stiffness coefficient of semi-infinite rod and the expressions for the interaction force history $R_{b}(t)$ in terms of convolution integral are given in Eq. (35) to Eq. (39). Here E is the elastic modulus of the soil, A is the cross-sectional area of the rod, $k_{g}$ is the elastic stiffness of the soil per unit length of the rod. The terms of $R_{b}(t)$ containing current (unknown) displacements $\left\{u_{b}^{t}\right\}_{n}$ and unknown velocities $\left\{\dot{u}_{b}^{t}\right\}_{n}$ will contribute to the stiffness $\left[K_{b b}^{g}\right]$ and $\left[C_{b b}^{g}\right]$ of the foundation-soil system (Eq. (18), Eq. (39). The remaining known terms of the convolution integral shall be calculated in ABAQUS using user-defined UAMP subroutine, and the corresponding amplitude is used to apply a concentrated force on mass $m_{0}$. Thus, the SSI system reduces to a fixed-base 2DOF dynamic system as shown in Figure 4 (b), where the ground motion is replaced by equivalent interaction force $R_{b}(t)$. The two degrees of freedom are the (total) displacements of the two masses $u_{s}^{t}$ and $u_{0}^{t}$. The numerical properties of the system are as detailed in Table 2 . In this case, the dynamics stiffness matrix of the foundation is a scalar value since there is only one DOF at the soil structure interface. The formulation is given by Eq. (35)-Eq. (39). 


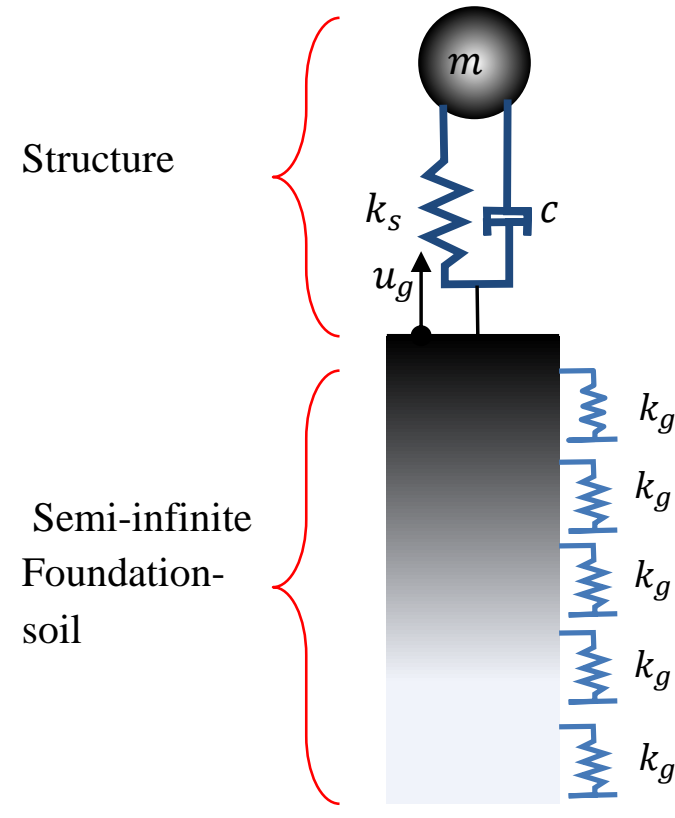

(a)

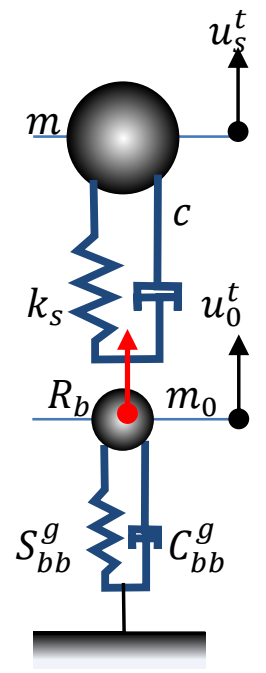

(b)

Figure 4. (a) and (b) 2DOF representing mass attached to a semi-infinite rod on elastic foundation

$S\left(a_{0}\right)=K i \sqrt{\left(a_{0}^{2}-1\right)}$

where $K=\sqrt{E A k_{g}}$ and $a_{0}=\frac{\omega}{c_{l} \kappa}, \kappa=\sqrt{\frac{k_{g}}{E A}}$

$S\left(a_{0}\right)=K i a_{0}+K i\left(\sqrt{\left(a_{0}^{2}-1\right)}-a_{0}\right)$

$S_{b b}(t)=K\left(\frac{\dot{\delta}(t)}{c_{l} \kappa}+\frac{J_{1}\left(t c_{l} \kappa\right)}{t}\right)$

So that , $K_{b b}^{g}=0, C_{b b}^{g}=\frac{K}{c_{l} \kappa}$ and $S_{r, b b}(t)=K \frac{J_{1}\left(t c_{l} \kappa\right)}{t}$

The system is subjected to an artificial time history, whose displacement and velocity histories are shown in Figure 5 (a). The system on being solved for the seismic response gives an accurate response as can be seen in Figure 5 (b). From the figure, it can also be observed that there is a permanent deformation caused by the elastic-perfect plastic nonlinearity of the structure spring. 
International Journal of Mathematical, Engineering and Management Sciences

Vol. 5, No. 3, 447-462, 2020

https://doi.org/10.33889/IJMEMS.2020.5.3.037

Table 2. Parameters for 2DOF system (Wolf, 1988)

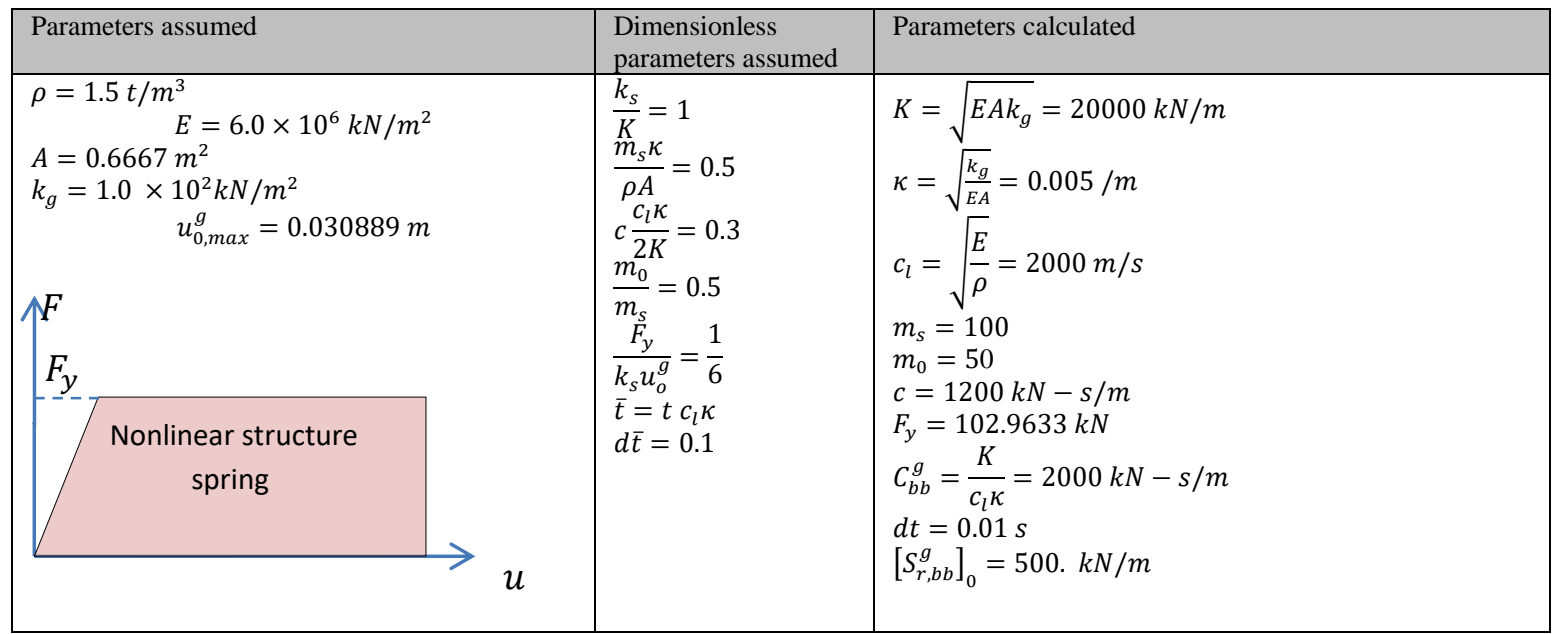

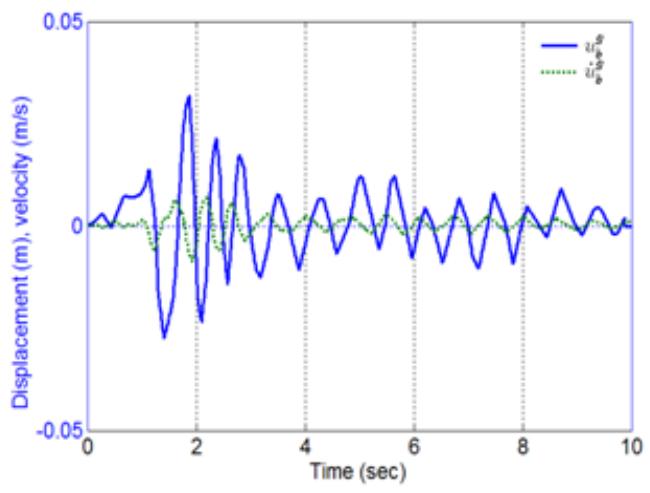

(a)

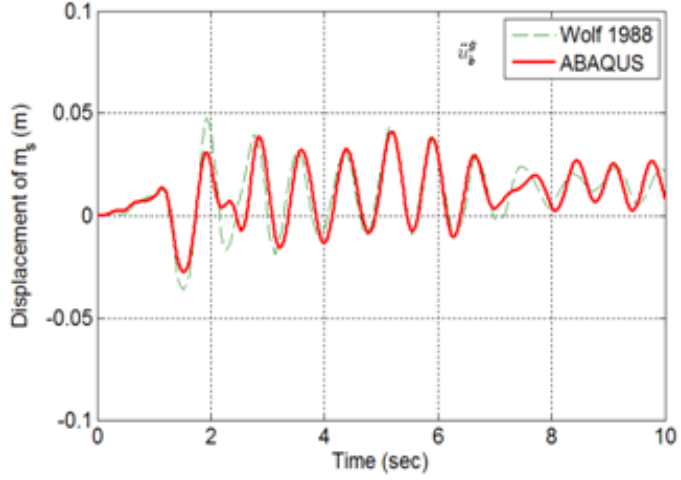

(b)

Figure 5. (a) Ground motion displacement and velocity, (b) comparison of displacement history of mass $m_{s}$ with benchmark result

\section{Analysis of 3DOF SSI System}

The system consists of a mass-spring-damper combination representing the dynamic properties of a structure, mounted on a rigid column of height ' $h$ '. This column is supported by a roller which represents a rigid ground in vertical direction. The horizontal translational and rotational degrees of freedom at the bottom of the column structure are governed by the corresponding components of foundation elasticity and damping. For the analysis purpose a foundation consisting of a rigid circular disk on an elastic half-space is considered. From the Figure 6, it can be noted that there are three degrees of freedom for the whole system- horizontal degree of freedom of the structure mass ' $\mathrm{m}$ ', the horizontal and rotational degrees of freedom of the foundation. The input values of the parameters and the expressions used to calculate the derived input values are shown in Table 3 . Here, $k_{s}$ is the stiffness of the structure spring, $\zeta$ is the structural damping ratio, $m$ is the 
International Journal of Mathematical, Engineering and Management Sciences

Vol. 5, No. 3, 447-462, 2020

https://doi.org/10.33889/IJMEMS.2020.5.3.037

structure mass, $a$ is the radius of the rigid circular disk foundation, $G, \rho, v, \zeta_{g}$ are the shear moduli, mass density, Poisson ratio, damping ratio of the soil respectively.

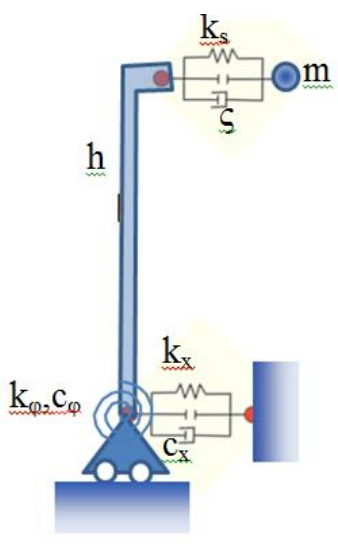

Figure 6. Three-DOF dynamic system representing SSI

The stiffness and damping values of foundation-soil system are obtained for a rigid circular disk of radius ' $a$ ', resting on a ground/ soil having a shear modulus of ' $G$ ', density of ' $\rho$ ', Poisson ratio of ' $v$ ', material damping constant of ' $\mathrm{g}$ ' 'This procedure is described in (Wolf, 1985) and the coefficients are shown in Figure 7 for horizontal and rocking components.

High frequency behaviour of rigid circular base mat resting on the surface of a layered half-space are (Wolf, 1988)

Horizontal: $\quad S_{b b, x}^{\infty}\left(a_{0}\right)=\pi G a i a_{0}$

Rocking : $\quad S_{b b, \phi}^{\infty}\left(a_{0}\right)=\frac{\pi G}{4} \sqrt{\frac{2(1-v)}{1-2 v}} a^{3} i a_{0}$

This forms the singular part of the dynamic stiffness coefficient. The regular part of the dynamic stiffness coefficient of a rigid circular disk or base mat resting on an undamped half-space can be obtained by separating the singular part from the dynamic stiffness coefficients shown in Figure 7.

From the singular part of dynamic stiffness coefficients the initial value (i.e., at $t=0+$ ) of the coefficient in time domain can be obtained. The regular part of dynamic stiffness coefficient is transformed to time domain using numerical Fourier transform to get $S_{r, b b}^{g}(t)$. The system can then be modelled in ABAQUS into a three DOF system shown in Figure 8. It is subjected to artificial earthquake ground motion described in the previous section (Figure 5). The results are shown in Figure 9 and Figure 10. Figure 9 gives the displacement history at base and structure mass, as well as the rotation at the base. It can be seen that the displacement response is considerably reduced with respect to the (free-field) ground motion in Figure 5. This is due to the high stiffness of soil. Further, the displacement of structural mass is quite magnified from that at 
International Journal of Mathematical, Engineering and Management Sciences

Vol. 5, No. 3, 447-462, 2020

https://doi.org/10.33889/IJMEMS.2020.5.3.037

the base. This is bound to happen for the selected $\bar{S}=0.1$, which means that the structure is quite flexible compared to the foundation stiffness. It can also be seen that the rotation of the base is quite negligible.

Table 3. Parameters defining the 3DOF dynamic system

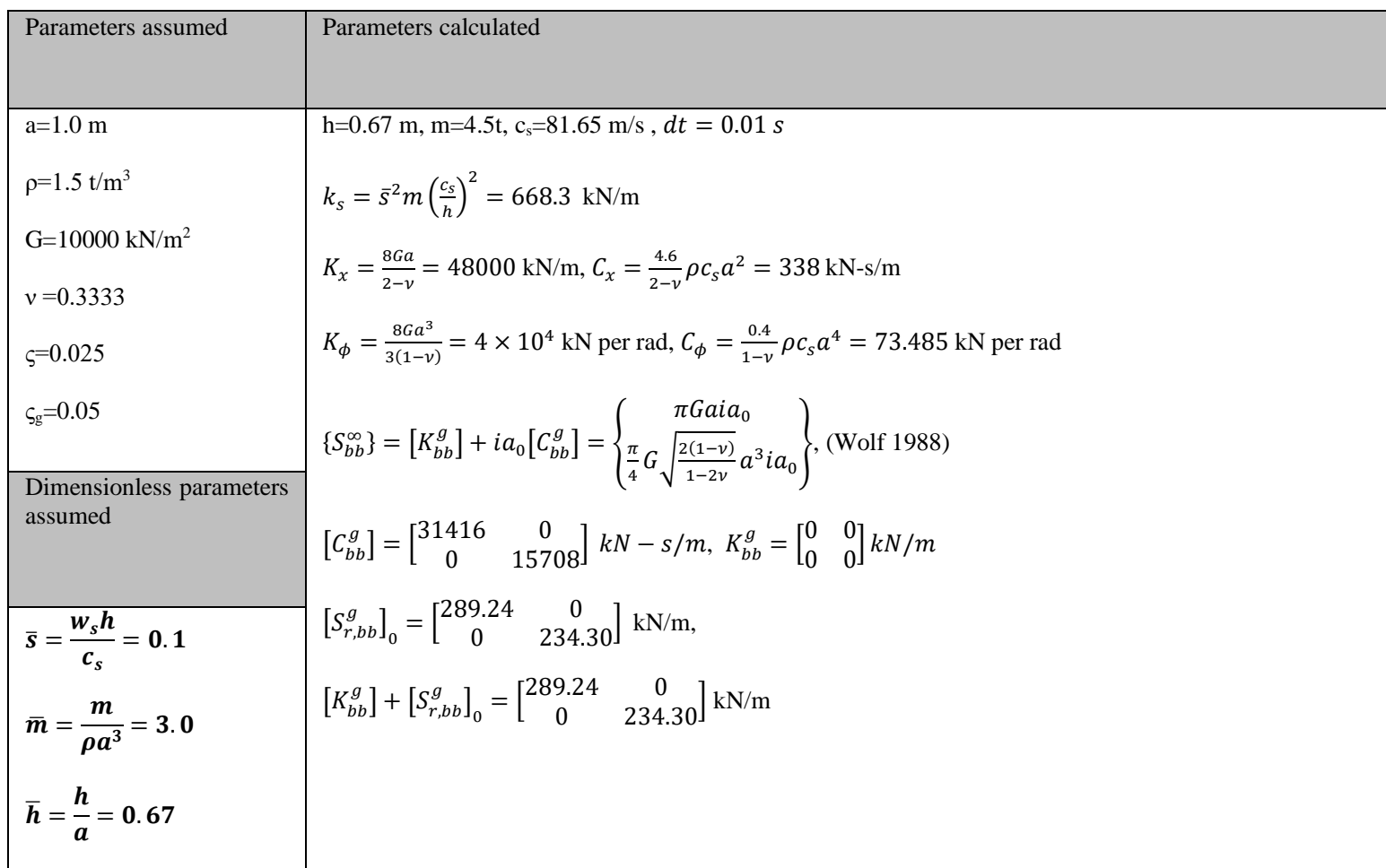

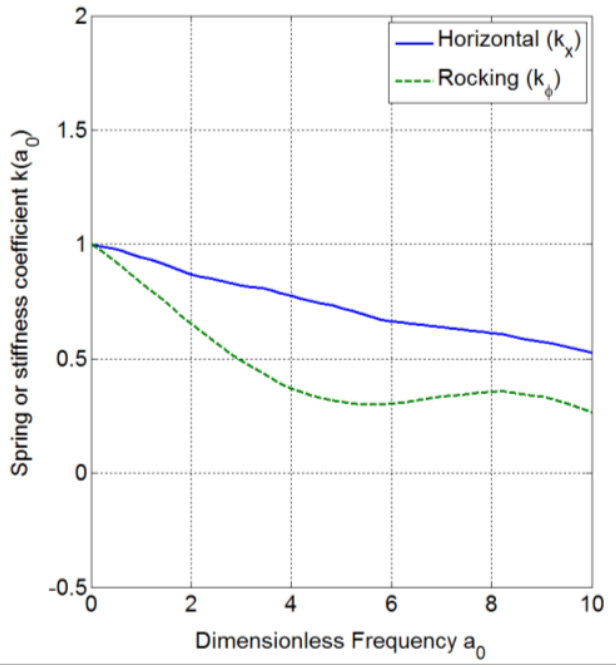

(a)
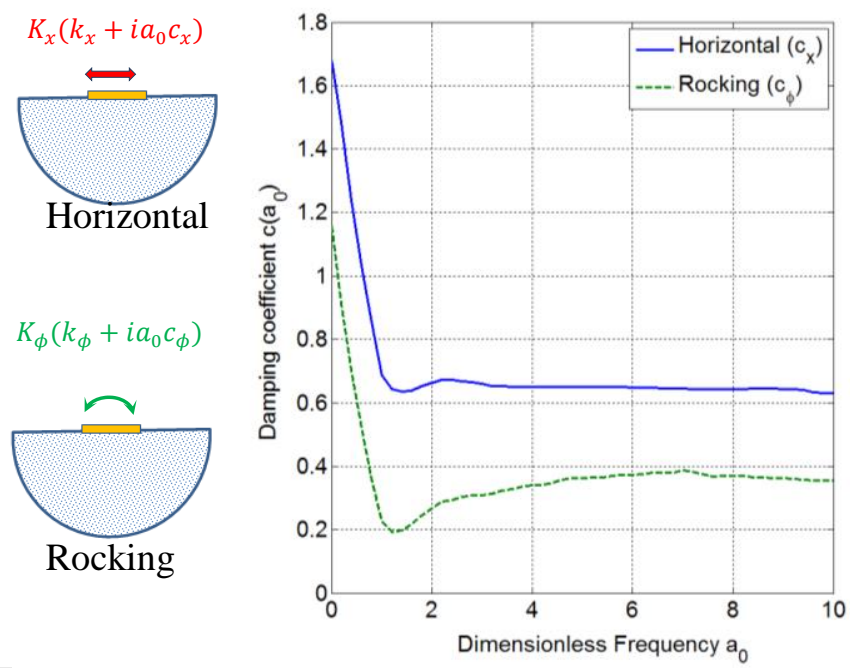

(b)

Figure 7. (a) and (b) Dynamic stiffness coefficients of rigid disk on half-space: spring \& damping 
International Journal of Mathematical, Engineering and Management Sciences

Vol. 5, No. 3, 447-462, 2020

https://doi.org/10.33889/IJMEMS.2020.5.3.037

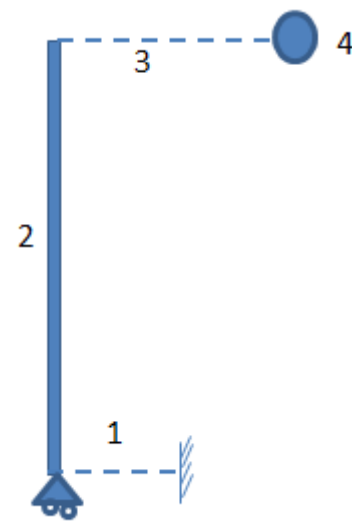

1. Axial + Rotational spring- CONN3D2

2. Rigid link - MPC Beam

3. Translator- CONN3D2

4. Structure Mass-MASS

Figure 8. ABAQUS model of the dynamic system

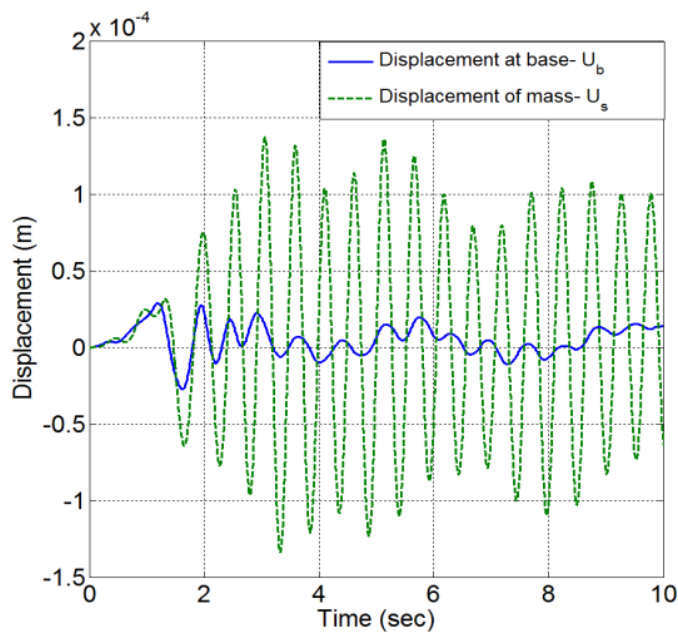

(a)

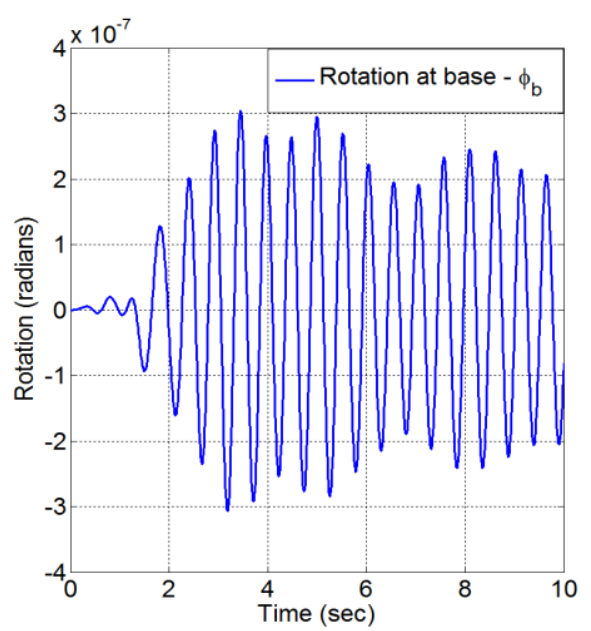

(b)

Figure 9. (a) and (b) Displacement time history of the 3DOF SSI system under EQ loading

The internal force in structural connector is shown in Figure 10 (b) and the applied force at the base in shown in Figure 10 (a). The applied force is the interaction force calculated using the convolution integral through the UAMP subroutine. The structure-connector being quite flexible in relation to the foundation experiences very low internal force as can be seen in Figure 10. 
International Journal of Mathematical, Engineering and Management Sciences

Vol. 5, No. 3, 447-462, 2020

https://doi.org/10.33889/IJMEMS.2020.5.3.037

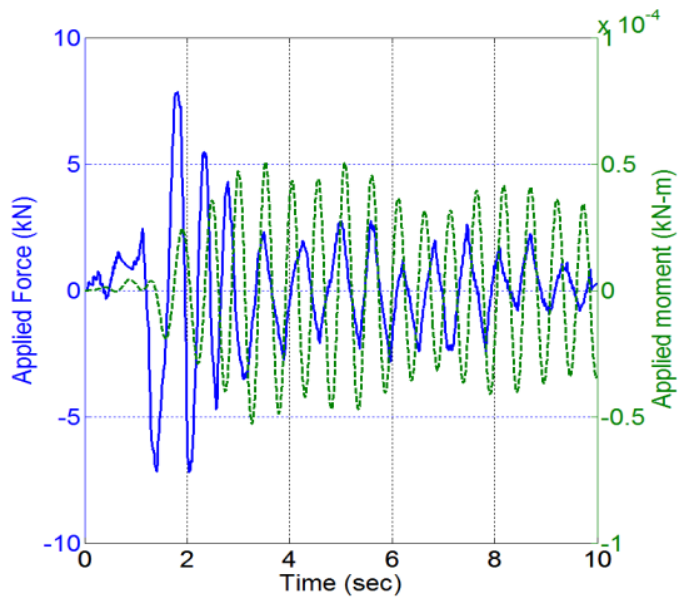

(a)

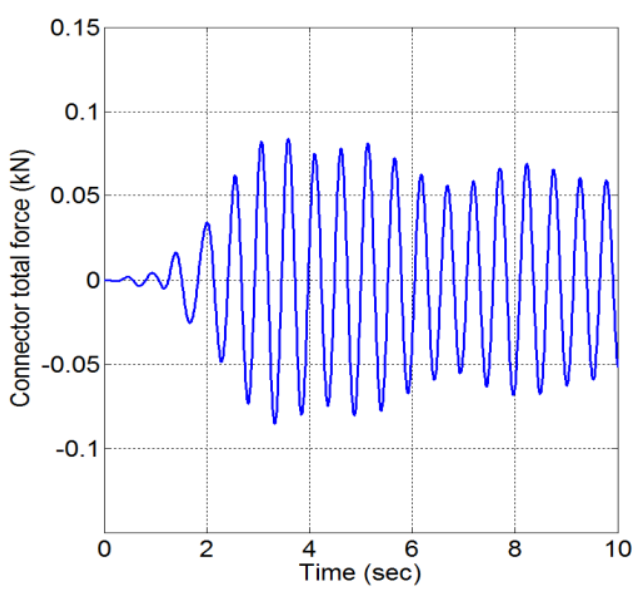

(b)

Figure 10. (a) Applied Force history at the base and (b) total force in Structure connector

\section{Conclusions}

The paper successfully presents steps for carrying out seismic analysis of three different SSI systems in time domain using ABAQUS software, wherein the user defined subroutine UAMP is programmed to interactively evaluate the soil structure interaction forces that ensure realistic transmitting boundaries. The procedure presented in the paper can be applied to continuum SSI models also.

\section{Conflict of Interest}

The authors confirm that there is no conflict of interest to declare for this publication.

\section{Acknowledgements}

The authors would like to express their sincere thanks to the editor and anonymous reviews for their time and valuable suggestions. This research was supported by Board of Research in Nuclear Sciences (BRNS), Department of Atomic Energy (DAE), Government of India, [No. 2012/36/49-BRNS]

\section{References}

Emani, P.K., \& Maheshwari, B.K. (2009). Dynamic impedances of pile groups with embedded caps in homogeneous elastic soils using CIFECM. Soil Dynamics and Earthquake Engineering, 29(6), $963-$ 973.

Emani, P.K., \& Maheshwari, B.K. (2010). Effectiveness of hybrid methods in analyzing frequencydependent systems with limited nonlinearity. International Journal of Advanced Computing, 2(2), 6067.

Kramer, S.L. (1996). Geotechnical earthquake engineering. Prentice Hall Upper Saddle River 569. 
International Journal of Mathematical, Engineering and Management Sciences

Vol. 5, No. 3, 447-462, 2020

https://doi.org/10.33889/IJMEMS.2020.5.3.037

Maheshwari, B.K., \& Emani, P.K. (2015). Three-dimensional nonlinear seismic analysis of pile groups using FE-CIFECM coupling in a hybrid domain and HISS plasticity model. International Journal of Geomechanics, 15(3), 04014055.

Poul, M.K., \& Zerva, A. (2018). Time-domain PML formulation for modeling viscoelastic waves with Rayleigh-type damping in an unbounded domain: theory and application in ABAQUS. Finite Elements in Analysis and Design, 152, 1-16.

Sarkar, R., \& Maheshwari, B. (2012). Effect of soil nonlinearity and liquefaction on dynamic stiffness of pile groups. International Journal of Geotechnical Engineering, 6(3), 319-330.

Solberg, J.M., Hossain, Q., \& Mseis, G. (2016). Nonlinear time-domain soil-structure interaction analysis of embedded reactor structures subjected to earthquake loads. Nuclear Engineering and Design, 304, $100-124$.

Spyrakos, C.C., \& Xu, C. (2003). Seismic soil-structure interaction of massive flexible strip-foundations embedded in layered soils by hybrid BEM-FEM. Soil Dynamics and Earthquake Engineering, 23(5), 383-389.

Wang M., Yang J. (2013). Three dimensional implementation of HISS model in ABAQUS. In: Yang Q., Zhang JM., Zheng H., Yao Y. (eds) Constitutive Modeling of Geomaterials. Springer Series in Geomechanics and Geoengineering. Springer, Berlin, Heidelberg.

Wegner, J.L., Yao, M.M., \& Zhang, X. (2005). Dynamic wave-soil-structure interaction analysis in the time domain. Computers \& Structures, 83(27), 2206-2214.

Wolf, J.P. (1985). Dynamic Soil-Structure Interaction, Prentice Hall, Inc, New Jersey.

Wolf, J.P. (1988). Soil-structure-interaction analysis in time domain. Prentice-Hall, Englewood Cliffs, New Jersey.

Wolf, J.P., \& Song, C. (1996). Finite Element Modelling of Unbounded Domain. Chichester: Prentice Hall, Inc. 